ISSN: 2600-5859

\title{
Estrategias metodológicas para mantener la atención de los estudiantes durante las clases de inglés
}

\section{Methodological strategies to keep the student's attention during English classes}

Mónica Paulina Castillo Niama. ${ }^{1}$, Silvana Patricia Célleri Quinde. ${ }^{2}$, Byron Rafael Soria Guerrero. ${ }^{3}$ \& Mayra Rosana Rojas Yumisaca. ${ }^{4}$

\section{Recibido: 05-12-2019 / Revisado: 27-12-2019 / Aceptado: 06-01-2020 / Publicado: 07-02-2020}

\section{Abstract \\ DOI: $\quad$ https://doi.org/10.33262/concienciadigital.v3i1.1.1144}

The objective of this study was to identify the main causes for the lack of attention of the Industrial Mechanic career students at Escuela Superior Polytechnical de Chimborazo in the English as a Foreign Language Classroom and at the same time to propose methodological strategies which could contribute to solve this problem. This was a qualitative research, the inquiry process was inductive, and the data collection instruments were the survey, which was directed to the entire population of this research and literature review. The population was based on 59 students in Level 1 who belong to the first and second English classrooms. There are three main causes why the teachers are not able to keep students' attention, they are monotonous classes, lack of motivation, and lack of concentration; thus, seven methodological strategies are suggested. Furthermore, three previous researchers related to the topic were analyzed; these studies support the present research, emphasize the problem importance and contribute with strategies and activities to be implemented in the English classrooms into the teaching and learning process. It is recommended that the

\footnotetext{
${ }^{1}$ Escuela Superior Politécnica de Chimborazo, Facultad de Mecánica. Riobamba, Ecuador, monicap.castillo@espoch.edu.ec

${ }^{2}$ Escuela Superior Politécnica de Chimborazo, Facultad de Administración de Empresas. Riobamba, Ecuador, patricia.celleri@espoch.edu.ec

${ }^{3}$ Escuela Superior Politécnica de Chimborazo, Facultad de Administración de Empresas. Riobamba, Ecuador, byron.soria@espoch.edu.ec

${ }^{4}$ Unidad Educativa Combatientes de Tapi, Área de Lengua Extranjera. Riobamba, Ecuador, rosana.rojas@educacion.gob.ec
} 
ISSN: 2600-5859

authorities pay attention not only in the student, but also in the teachers' needs, so that the planned learning objectives could be reached.

Keywords: Attention, motivation, strategies, English, qualitative

\section{Resumen}

El objetivo del presente trabajo fue identificar las principales causas de la falta de atención de los estudiantes de la Carrera de Mecánica Industrial de la Escuela Superior Politécnica de Chimborazo durante las clases de inglés como Lengua Extranjera y a la vez proponer estrategias metodológicas que contribuyan a resolver este problema. Se trató de una investigación de tipo cualitativo, el proceso de indagación fue inductivo y los instrumentos de recolección de datos fueron la encuesta, la cual fue dirigida a toda la población de esta investigación y la revisión bibliográfica. La población estuvo compuesta por 59 estudiantes de Nivel 1 de inglés de los paralelos 1 y 2. Se identifican tres causas fundamentales por las cuales el docente no logra mantener la atención de los estudiantes, estas son: clases monótonas, falta de motivación y falta de concentración; a lo que se sugieren siete estrategias metodológicas. Adicionalmente, se analizan tres estudios previos sobre el tema, mismos que respaldan el presente estudio, enfatizan la importancia del problema y aportan con estrategias y actividades que pueden ser implementadas dentro del aula de inglés en el proceso enseñanza aprendizaje. Se recomienda que se brinde la atención necesaria no solamente a las necesidades de los estudiantes, sino también de los docentes, a fin de que se puedan alcanzar los objetivos de aprendizaje planteados.

Palabras claves: Atención, motivación, estrategias, inglés, cualitativo

\section{Introducción}

Uno de los problemas que se presentan en el aula de inglés como lengua extranjera, es que la enseñanza ha venido siendo memorística, monótona y nada práctica, lo cual no ha permitido que los estudiantes relacionen la teoría con la práctica y su entorno al desarrollar las habilidades básicas de un segundo idioma como son: escuchar, hablar, leer y escribir. Es decir, permitir a los estudiantes tener un pensamiento crítico al identificar y resolver problemas que se presentan en la sociedad. Por lo tanto, depende mucho de la habilidad que tengan los docentes para usar estrategias metodológicas, el mantener la atención de los estudiantes y facilitar el aprendizaje de un nuevo 
ISSN: 2600-5859

idioma en ambientes motivadores que generen aprendizaje y permitan a los estudiantes tener una zona de confort. (Cedeño \& Ochoa, 2019)

Las estrategias metodológicas son de suma importancia en el quehacer educativo; por lo tanto, esta investigación se encamina a proponer estrategias metodológicas en el proceso de enseñanza aprendizaje del idioma inglés como Lengua Extrajera, para mantener la atención de los estudiantes durante las clases y fortalecer las practicas educativas de los docentes que imparten esta asignatura, a fin de convertir cada aula de clase un lugar cómodo y agradable para enseñar y aprender. (Arguello \& Sequeira, 2016)

Es así, que existen trabajos de investigación que constituyen una importante contribución a entender las posibles causas y soluciones de la falta de atención de los estudiantes.

El primer aporte corresponde a Tipán (2017), quien manifiesta que uno de los mayores problemas que enfrentan los maestros de inglés es el bajo nivel de atención de los estudiantes (Tipán, 2017). Al finalizar su estudio la autora concluye que los comics son una estrategia metodológica efectiva para mantener la atención de los estudiantes en la clase de inglés; sin embargo, no se utiliza de manera constante.

En el estudio titulado "Estrategias activas para la enseñanza del idioma inglés como lengua extranjera a los estudiantes de la Universidad Central del Ecuador," los autores manifiestan que la falta de motivación para llevar a cabo un proceso de aprendizaje participativo es un problema generalizado en el aula de inglés, ya que este es simplemente considerado como un complemento de la formación profesional del estudiante. Se concluye que las estrategias de enseñanza constituyen una herramienta para que el estudiante se involucre de forma activa, pueda manejar de manera autónoma su aprendizaje; y se recomienda que el docente planifique actividades como trabajo en equipo, diálogos en pares y dramatizaciones para poder mantener la atención de los estudiantes durante la clase de inglés. (Vélez \& Ramos, 2018)

Pila (2012), indica que existen varios factores que han afectado el proceso de enseñanza aprendizaje; entre los cuales resaltan: el desconocimiento de la importancia de dominar el idioma inglés como segunda lengua, la falta de motivación en el estudiante, la aplicación de métodos y 
ISSN: 2600-5859

estrategias tradicionales y la falta de atención de los alumnos. Ante lo cual concluye que se necesita que el docente desarrolle prácticas motivacionales como trabajos en grupos o en parejas, canciones, juegos, y dinámicas que despierten el interés y atención del estudiante. (Pila, 2012, pág. 121)

Adicionalmente a los hallazgos mencionados, en la presente investigación se identificaron tres causas que originan la falta de atención de los estudiantes durante el desarrollo de las clases de inglés, estas son: falta de motivación por parte de los docentes al no indicar cuales son las principales ventajas de aprender otro idioma como es el inglés; clases monótonas y falta de concentración de los estudiantes.

Ibarra (2018), puntualiza que todo resultado de aprendizaje debe ser evaluado y esa avaluación debe estar diseñada a fin de que permita examinar si el estudiante logro o no el aprendizaje comprometido, pero para ello se requiere estrategias las cuales permitan ubicar al estudiante donde pueda demostrar o evidenciar en qué medida logró el aprendizaje. Es decir que, la elección de estrategias metodológicas para mantener la atención de los estudiantes durante las clases de inglés debe ser un aporte muy importante por parte del docente con el fin de obtener los logros de los resultados de aprendizaje. (Ibarra, 2018)

El compromiso de los docentes de la Escuela Superior Politécnica de Chimborazo, es mejorar la calidad de educación de los estudiantes dentro del proceso enseñanza aprendizaje de inglés como Lengua Extranjera, aportando con claridad a la buena formación académica de los estudiantes, promoviendo la integración y consistencia, pero esto no se logrará si no existe la motivación adecuada, concentración constante en las clases y si los docentes no rompen las clases convencionales y monótonas donde no existe estrategias metodológicas para mantener la atención de los estudiantes.

Con este trabajo se beneficiará a la comunidad politécnica, a los estudiantes ya que ellos son los principales protagonistas del proceso educativo y a los docentes, quienes podrán poner en práctica las estrategias metodológicas sugeridas a fin de lograr resultados óptimos en el aprendizaje de inglés como Lengua Extranjera. 


\section{Revisión Literaria}

\section{Estrategias metodológicas}

Es cada vez más común escuchar a los docentes de inglés, lo difícil que se ha tornado lograr que los estudiantes los escuchen y estén suficientemente atentos en el aula. Es así que, la falta de atención es uno de los principales desencadenantes de los retrasos en el aprendizaje. Las nuevas formas de ocio y de comunicación, como los teléfonos móviles e Internet, son fuentes de estímulos constantes que crean una necesidad de respuesta rápida, lo que favorece a que los estudiantes se distraigan tanto dentro como fuera del aula. (Belver, 2013)

Por esta razón, en las aulas de clases muchos docentes han puesto en práctica diferentes estrategias metodológicas; un conjunto de técnicas y procedimientos esenciales e imprescindibles en el proceso de enseñanza aprendizaje, con el firme propósito de facilitar un espacio donde los estudiantes puedan desarrollar sus habilidades y destrezas para construir aprendizajes significativos. (Cruz, Criollo, \& Raffo, 2017)

Considerando la importancia de implementar estrategias metodológicas efectivas para mantener la atención de los estudiantes en el aula de inglés, a continuación, se presentan algunos de los hallazgos más relevantes que contribuirán a erradicar este problema se hace más frecuente en el proceso de enseñanza aprendizaje.

1. Dar protagonismo a la parte afectiva y comunicativa. Los docentes de nivel superior desafortunadamente no han hecho consciencia de la importancia que tiene mantener una relación harmónica con los estudiantes durante el proceso de enseñanza aprendizaje; dejando de lado la parte afectiva; sin considerar que la falta de atención podría deberse a que la mente del estudiante está ocupada en un problema de tipo persona. En este sentido, la comunicación y confianza es la primera estrategia que se debe poner en práctica dentro de la clase de inglés. (Universidad Internacional de Valencia, 2018)

2. Incentivar la participación de los alumnos. Los resultados del presente estudio demuestran que una de las causas de la falta de atención es que las clases son monótonas; por lo que el docente debe escoger contenidos interesantes y utilizar aplicaciones como 
ISSN: 2600-5859

Kahoot que despierten el interés de los estudiantes y hagan que las clases de inglés sean más divertidas. (Sánchez, 2018)

3. Empezar con un calentamiento mental. Se recomienda que el docente planifique actividades de resolución de problemas o desafío; por ejemplo, comparar, adivinar; resolver crucigramas, sopa de letras, cruzadas y sudokus. (Universaria, 2016)

4. Introducir cambios con ciclos y pausas. De acuerdo con Tokuhama (2011) la capacidad de mantener la atención sostenida varía entre 10 y 20 minutos, por lo que se debe utilizar bloques que no superen los 15 minutos y de esa manera incrementar la capacidad atencional de los estudiantes; facilitando así el procesamiento y consolidación de la información (Tokuhama, 2011).

5. Desplegar esporádicamente una pregunta o un desafío. A fin de monitorear el nivel de atención de los estudiantes, se puede realizar preguntas sobre el tema que se está estudiando y también evaluar su la comprensión del mismo utilizando herramientas como Socrative, que además proporciona una retroalimentación rápida y efectiva. (Teyssier, 2017)

6. Utilizar el humor. Hablar de humor en el ámbito educativo, no es habitual. Estudiosos de este tema afirman que a pesar de que el humor ha ganado prestigio en ámbitos culturales, aún sigue al margen de las aulas. Berger (1999), afirma que la experiencia cómica tiene una influencia positiva en el aprendizaje (Berger, 1999), pues favorece el desarrollo de la creatividad y el pensamiento reflexivo en los estudiantes; entonces, los docentes deben comprender que la risa es un elemento esencial para el aprendizaje. (Adams, 2012)

7. Diseñar tareas auténticas y experiencias de aprendizaje. Dentro de los hallazgos de la presente investigación, se identificó que existe falta de motivación por parte de docentes, lo que contribuye a que no se pueda retener la atención de los estudiantes durante las clases de inglés. Por ello, se debe incrementar actividades auténticas dentro y fuera del aula. Wiggins (1998), considera que una actividad auténtica se caracteriza por reproducir el conocimiento en situaciones reales. Las actividades auténticas y experiencias de aprendizaje incluyen: ferias, casas abiertas, ejecución de un festival de música o teatro y participación en debates sobre asuntos sociales (Wiggins, 1998 ). El rol del estudiante es 
ISSN: 2600-5859

planear, diseñar y construir, lo cual permite que la clase ya no se centre en el profesor. (Rodriguez, 2018)

Finalmente, Sztabnik (2017) explica que los más importante es ganarse la atención de los estudiantes al inicio y al final de la clase; ya que cada momento de la clase importan, pero hay ocho minutos fundamentales que son los dedicados al principio y el final de una lección; si ésta no comienza fuerte (Sztabnik, 2017), el interés del estudiante disminuye y el docente deberá hacer grandes esfuerzos para atrapar la atención de los estudiantes; y si no se comprueba lo que han entendido, resultará complicado evaluar si se ha conseguido el objetivo de la lección. Para realizar mantener la magia en estos ocho minutos se recomienda herramientas tales como: YouTube, Exit Tickets, Twitter, Pinterest y WhatsApp. (Arenas, 2019)

\section{Atención}

En la publicación titulada "La Atención: Activando el Aprendizaje", se puntualiza que la atención es un mecanismo que posee el ser humano, la misma que permitirá discriminar, entre los estímulos que percibe y que el nivel de atención no debe ir más allá de los límites máximos ya que producen cansancio, ni limites mínimos que producirán aburrimiento. (Tortolero, 2018)

\section{Características de la Atención}

Las características más importantes de la atención son:

- Amplitud. Hace referencia a la cantidad de información que se puede atender a la vez y también al número de tareas que se realizan simultáneamente.

- Actividad. La atención supone un incremento en la actividad del sistema nervioso e intelectual del estudiante.

- Selectividad. La atención es selectiva es decir se refiere a las tareas que selecciona y hace que establezca jerarquías y prioridades filtrando de esta manera toda la información y seleccionando solo aquella que es relevante.

- Intensidad. Es la cantidad de atención que prestamos a una tarea u objeto.

- Control. Se caracteriza por ser una de las funciones más importantes de la atención ya que responden a objetivos y requieren de respuestas determinadas. El control de la atención es dirigir el pensamiento y la acción hacia una meta. (Tortolero, 2018) 
- García (2018) manifiesta que existen los siguientes tipos de atención:

Atención Interna y Externa.

Atención Abierta y Encubierta.

Atención Voluntaria e Involuntaria.

Atención Visual y Atención Auditiva.

Atención Selectiva, Atención Dividida y Atención Sostenida. (García, 2018)

Todos estos tipos de atención son características descriptivas e individuales que presentan las personas bajo determinada circunstancia.

\section{Objetivos}

\section{Objetivo General}

- Proponer estrategias metodológicas para mantener la atención de los estudiantes durante las clases de inglés.

\section{Objetivos Específicos}

- Analizar los hallazgos de estudios previos relacionados con la falta de atención.

- Establecer las causas que originan la falta de atención en los estudiantes del Nivel A1 de inglés.

- Presentar estrategias metodológicas encaminadas a mejorar la atención de los estudiantes durante las clases de inglés.

\section{Metodología}

Por la naturaleza de los datos, la presente investigación se enmarca en el método cualitativo pues se estudia la falta de atención de los estudiantes durante la clase de inglés como una realidad social dentro del entorno de la enseñanza. Se centra en los sujetos de estudio, 59 estudiantes de la carrera de Mecánica Industrial del Nivel A1 de inglés de los paralelos 1 y 2 . Asimismo, es un proceso de indagación inductivo en el cual, los investigadores interactúan con los participantes a través de una entrevista, en la búsqueda de una solución al problema detectado; es por lo cual, el principal objetivo de la presente investigación es proponer estrategias metodológicas para mantener la atención de los estudiantes durante las clases. También es importante mencionar que el método 
ISSN: 2600-5859

cualitativo no descubre, sino que construye el conocimiento en base al comportamiento entre las personas implicadas y su conducta observable. En el caso del presente estudio, se pudo evidenciar el bajo rendimiento de los estudiantes en la asignatura de inglés; por tal motivo se aplicó una encuesta en la cual los participantes afirman que el profesor no consigue mantener la atención de los estudiantes durante las clases; posteriormente, con los datos obtenidos de la encuesta, se procede a realizar una exhaustiva revisión bibliográfica a fin de sugerir estrategias efectivas para mantener la atención de los estudiantes durante las clases de inglés.

En cuanto al diseño, se trata de una investigación diagnóstica; ya que, los investigadores se enfocan hacia la evaluación de la causa raíz de la falta de atención de los estudiantes. En este diseño de investigación también se evalúan los elementos que contribuyen a una situación problemática. Y está compuesto de tres partes: 1) Inicio del problema; 2) Diagnóstico; y 3) Solución. (Maldonado, 2015)

La población estuvo conformada por los estudiantes de la Carrera de Mecánica Industrial de la Escuela Superior Politécnica de Chimborazo; se seleccionó toda la población por conveniencia, puesto que los estudiantes de la Carrera de Mecánica Industrial ya estuvieron agrupados previamente y los investigadores podían acceder a información de los mismos fácilmente.

$\mathrm{Al}$ inicio de la presente investigación se identificó el problema, por medio de la aplicación de un cuestionario, que estuvo conformado por 10 preguntas cerradas de escala numérica, donde se presentaron dificultades de aprendizaje comúnmente observados en el aula de inglés que interfieren directamente en los logros de aprendizaje y rendimiento académico de los estudiantes.

Posteriormente, se desarrolló una entrevista, con la finalidad de conocer las posibles causas de la falta de atención de los estudiantes, para lo cual los investigadores establecieron un horario, a fin de poder recabar esta valiosa información directamente desde la fuente y de manera individual con los 59 estudiantes de la Carrera de Mecánica Industrial.

Finalmente, se realizó una exhaustiva revisión literaria sobre el tema a fin de poder sugerir alternativas de solución, basadas en estrategias metodológicas que puedan ser fácilmente puestas 
ISSN: 2600-5859

WWW.concienciadig1tal.org
Vol. 3, $\mathrm{N}^{\circ} 1.1$, p. 237-252, febrero, 2020

en marcha dentro del proceso de enseñanza-aprendizaje y que coadyuven a mejorar el rendimiento académico de los estudiantes de la Carrera de Mecánica Industrial del Nivel A1 de inglés de la Escuela Superior Politécnica de Chimborazo.

\section{Resultados}

Gráfico 1: Encuesta de identificación del problema

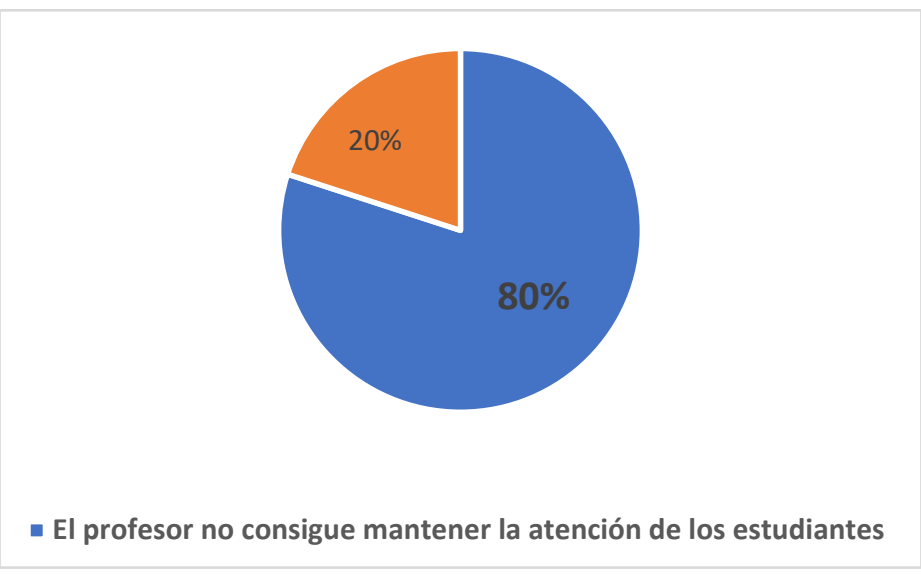

Fuente: Encuesta

Autor: Investigadores

Los resultados del cuestionario aplicado a la población involucrada en esta investigación demuestran que el principal problema es que el docente no consigue mantener la atención de los estudiantes durante las clases de inglés. Es decir, un $80 \%$ de estudiantes encuestados coinciden en sus respuestas, de que el profesor no mantiene ni el interés ni la atención de los estudiantes en las clases de inglés, lo cual limita el desarrollo adecuado de los estudiantes por la falta de interés que ponen en las aulas. Mientras que un $20 \%$ que es la gran minoría resalta que, si consiguen mantener la atención durante las clases de inglés, esto indica que este porcentaje de estudiantes si desarrollan su interés en las aulas.

Concluyendo, que la falta de atención en las aulas no permite que se desarrolle con éxito el proceso de enseñanza aprendizaje, ya que el profesor no consigue mantener la atención de los estudiantes 
ISSN: 2600-5859

a través de las actividades realizadas en clases de inglés; lo cual produce la falta de atención por parte de los estudiantes y por ende no permite un buen desarrollo del proceso enseñanza aprendizaje.

Tabla 1. Resultados de la entrevista

\section{Principales causas de la falta de atención}

1. Clases monótonas

2. Falta de motivación

3. Falta de concentración

Fuente: Encuesta

Autor: Investigadores

En cuanto a los resultados obtenidos de la entrevista que se aplicó a los 59 estudiantes de la carrera de Mecánica Industrial del Nivel A1 de los paralelos 1 y 2 de la Escuela Superior Politécnica de Chimborazo, sobre las posibles causas para la falta de atención de los estudiantes durante las clases de inglés, los resultados arrojaron que existen tres causas principales en un orden de prioridades. Este orden fue producto de los criterios de los estudiantes quienes coincidieron en que la falta de atención se debe a estas tres causas principales que son: clases monótonas por parte de los profesores, falta de motivación de los profesores y falta de concentración en las clases por parte de os estudiantes. Estos son los tres factores que no están permitiendo que las clases de inglés se desarrollen de la mejor manera y por lo tanto se ha convertido en un problema académico para el óptimo proceso de enseñanza aprendizaje del idioma inglés como lengua extranjera en los estudiantes.

\section{Discusión}

"Investigar es ver lo que todo el mundo ha visto, y pensar lo que nadie más ha pensado." Albert Szent-Gyorgyi. En esta investigación se muestra como un problema tan común como es la falta de atención de los estudiantes durante las clases de inglés, ha sido, de cierta manera ignorado o no se le ha dado la relevancia que merece dejando de lado la relevancia que deben dar los docentes a este problema. 
ISSN: 2600-5859

Como se observa en los resultados de la encuesta aplicada a los estudiantes de la Carrera de Mecánica Industrial, uno de los problemas más notables en el proceso de enseñanza aprendizaje de inglés como lengua extranjera es que el docente no logra mantener la atención de los estudiantes en clase y este hallazgo se respalda también con estudios previos como el de Tipán (2017), quien coincide en que existe un bajo nivel de atención por parte de los estudiantes y recomienda el uso de comics como una posible solución al problema. Si bien la autora realiza su investigación con dos variables similares: bajo nivel de atención y enseñanza de inglés, la población que toma como base responde a estudiantes de novemos y decimos años de educación general básica; mientras que la presente investigación se enfoca en estudiantes de nivel superior, lo que podría influir en la efectividad de la estrategia sugerida; los comics. Sin embargo; antes de implementar esta estrategia los docentes deberían considerar los intereses y preferencias de los estudiantes antes de elegir el material con el que se va a trabajar (Tipán, 2017).

De igual manera, Vélez y Ramos (2018), en su artículo científico publicado en la revista Publicando, afirman que la falta de motivación en los estudiantes es un problema generalizado que está sucediendo en las aulas de inglés, pero que no existe una clara contribución para dar solución al problema que está latente en las aulas, por lo tanto esta es más bien una investigación de tipo descriptivo que se enfoca en identificar las causas del problema y la importancia de que el docente involucre la participación del estudiante durante el proceso de enseñanza-aprendizaje; se sugiere planificar actividades como trabajo en equipo, diálogos y dramatizaciones, pero al no contarse con una intervención, no se conoce la efectividad de estas estrategias; no obstante, las actividades recomendadas pueden añadirse a la lista propuesta en la presente investigación. (Vélez \& Ramos, 2018)

En lo referente al estudio de Pila (2012), se relaciona mayormente a las causas que coindicen con las del presente estudio y se considera como una valiosa contribución la necesidad de concienciar en nuestros estudiantes la importancia de dominar un segundo idioma como es inglés; es decir exponer todas las ventajas que tiene un individuo al saber el idioma inglés como lengua extranjera y además para que inglés no se la considera solamente como una asignatura más que se debe aprobar, sino como una valiosa herramienta que les abrirá muchas posibilidades en el campo 
ISSN: 2600-5859

profesional y ocupacional (Pila, 2012). En lo que respecta a las clases tradicionales, se debe considerar que, en muchas de las ocasiones, este estancamiento, se debe a que el docente no se siente motivado, pues considera que su trabajo no es valorado y respaldado; pues ni siquiera cuenta con un espacio adecuado para desarrollar sus actividades académicas, existe escasez de equipo tecnológico y, lo que, es más, la capacitación es muy limitada. A igual que Vélez y Ramos (2018), recomiendan trabajos en grupo o parejas, juegos, dinámicas y también añade canciones. Esto nos lleva a la conclusión de que el éxito está en la variedad, ahora es responsabilidad del docente poner en práctica cada una de las estrategias sugeridas en la presente investigación; sólo así se podrá dar solución a la falta de atención de los estudiantes de inglés como Lengua Extranjera.

\section{Conclusiones}

- Luego de realizar una breve revisión literaria descriptiva, se presentan siete estratégicas metodológicas que podrán ser implementadas dentro del aula de clases de inglés como Lengua Extranjera, con la finalidad de mejorar el nivel de atención de los estudiantes.

- Se analizaron tres estudios que no sólo tienen un elevado nivel de concomitancia con el presente estudio, sino que adicionalmente aportan con hallazgos muy válidos, como la necesidad de que los estudiantes entiendan la importancia de dominar una segunda lengua $\mathrm{y}$ de que los docentes cuenten con los herramientas, instrumentos, equipos y materiales que contribuyan a un mejor desarrollo de sus clases.

- Como se muestra en la Tabla 1, las principales causas de la falta de atención son: clases monótonas, falta de motivación y falta de concentración, las cuales coinciden con la información recabada de los estudios que se citan en la presente investigación.

- A más de las siete estrategias descritas en este trabajo, se añaden las mencionadas en los estudios previos como son: trabajos grupales y en parejas; comics, dinámicas, juegos, diálogos, dramatizaciones y canciones.

\section{Recomendaciones}

- Una vez finalizado el trabajo de investigación se realizan las siguientes recomendaciones: 
ISSN: 2600-5859

- Que tanto los docentes, con el apoyo de las autoridades institucionales desarrollen proyectos de investigación dentro del aula; pues problemas como la falta de atención son evidentes y es responsabilidad de todos quienes conformamos una institución educativa, dar solución a los mismos.

- Que los docentes implementen estrategias metodológicas encaminadas a mejorar el nivel de atención de los estudiantes, mediante la investigación acción; pues es una herramienta metodológica heurística para la comprensión y sobre todo la transformación de realidades y prácticas socio educativas.

- Que se concientice a las autoridades sobre las necesidades de los docentes de inglés, especialmente en lo relacionado a capacitación.

\section{Referencias Bibliográficas}

Adams, P. (2012). INED21. Obtenido de https://ined21.com/humor-y-educacion-unidos-por-larisa/

Arenas, V. (2019). School Market. Obtenido de https:/www.schoolmarket.es/ocho-minutos-paracaptar-la-atencion-de-los-alumnos/

Arguello, B., \& Sequeira, M. (2016). Estrategias metodológicas relacionadas a la enseñanza aprendizaje de la disciplina . Juigalpa: Universidad Autónoma de Nicaragua.

Belver, C. (2013). La importancia de motivar el interés en nuestros alumnos. Reflexión Académica , 155-157.

Berger, P. (1999). Sobre la anatomía de lo cómico. ASELE.

Cedeño, A., \& Ochoa, M. (2019). Las estrategias didácticas y su influencia en el aprendizaje significativo . Guayaquil: Univesidad Laica Vicente Rocafuerte.

Cruz, M., Criollo, M., \& Raffo, D. (2017). Estretegias metodológicas para la enseñanzaaprendizaje . INNOVA, 54-69.

García, F. (2018). Motivación, aprendizaje y rendimiento escolar . REME.

Hernández, R. (2002). Metodología de la investigación. México: MfGraw Hill. 
ISSN: 2600-5859

WWW.concienciadig1tal.org

Vol. 3, $\mathrm{N}^{\circ} 1.1$, p. 237-252, febrero, 2020

Ibarra, P. (2018). Manual de orientaciones estratégicas metodológicas de enseñanza. Temuco: Universidad de la Frontera.

Pila, J. (2012). La motivación como estrategia de aprendizaje en el desarrollo de competencias comunicativas . Guayaquil: Universidad de Guayaquil.

Rodriguez, K. (2 de Octubre de 2018). EducaciónBlog.com. Obtenido de https://koryrodriguez.com/educacion/actividades-autenticas/

Sztabnik, T. (2017). Atención y aprenizaje . Aula.

Teyssier, M. (14 de Marzo de 2017). Educación Hoy. Obtenido de http://www.gruposm.com.mx/?q=blog-estrategias-para-mejorar-la-atencion-de-los-alumnos-en-el-aula

Tipán, M. (2017). El cómic como estrategia en la atención de los estudiantes de inglés. Quito: Universidad Central del Ecuador.

Tokuhama, T. (2011). ¿Qué puede hacer la ciencia por la enseñanza y apendizaje? Vespertino.

Tortolero, B. (2018). Psicopedia . Obtenido de https://psicopedia.org/7179/atencion-procesoactivo-del-aprendizaje/

Universaria. (5 de Septiembre de 2016). REBID. Obtenido de http://noticias.universia.pr/educacion/noticia/2016/09/05/1143275/como-tomar-apuntesclase-metodo-cornell.html

Universidad Internacional de Valencia. (21 de Marzo de 2018). Obtenido de https://www.universidadviu.com/algunas-ideas-para-mantener-la-atencion-de-losalumnos/

Vélez, D., \& Ramos, I. (2018). Estrategias activas para la enseñanza del idioma inglés como lengua extranjera a los estudiantes de la Universidad Central del Ecuador. Revista Publicando , 490-506.

Wiggins, G. (1998 ). Brindado devolución continua . San Francisco : Educational Assessment.

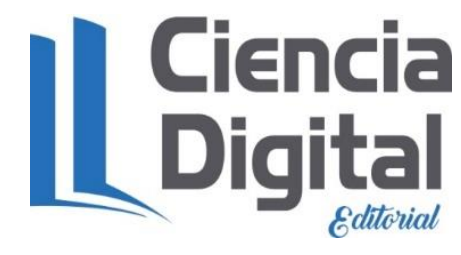




\section{PARA CITAR EL ARTÍCULO INDEXADO.}

Castillo Niama, M. P., Célleri Quinde, S. P., Soria Guerrero, B. R., \& Rojas Yumisaca, M. R. (2020). Estrategias metodológicas para mantener la atención de los estudiantes durante las clases de inglés. ConcienciaDigital, 3(1.1), 237-252. https://doi.org/10.33262/concienciadigital.v3i1.1.1144

El artículo que se publica es de exclusiva responsabilidad de los autores y no necesariamente reflejan el pensamiento de la Revista Conciencia Digital.

El artículo queda en propiedad de la revista y, por tanto, su publicación parcial y/o total en otro medio tiene que ser autorizado por el director de la Revista Conciencia Digital.
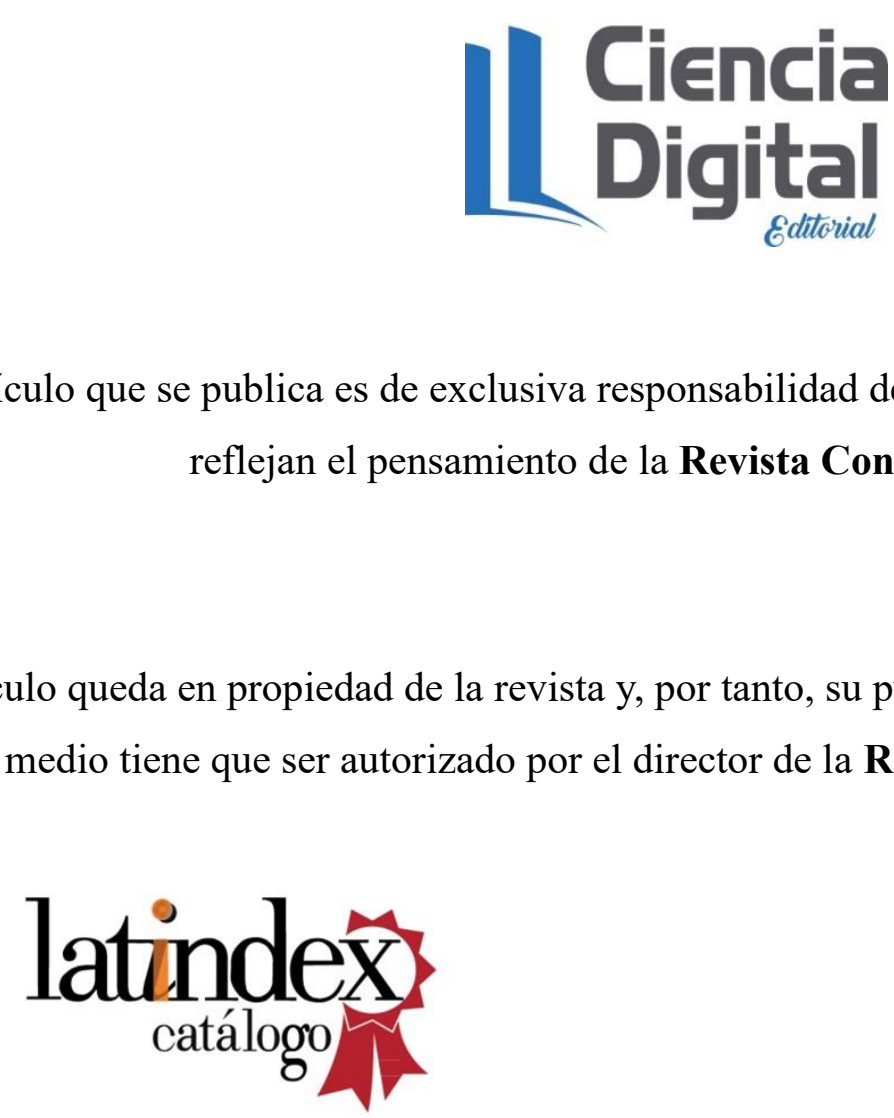